\title{
RICCI FLAT CALABI'S METRIC IS NOT PROJECTIVELY INDUCED
}

\author{
ANDREA LOI, MICHELA ZEDDA, AND FABIO ZUDDAS
}

\begin{abstract}
We show that the Ricci flat Calabi's metrics on holomorphic line bundles over compact Kähler-Einstein manifolds are not projectively induced. As a byproduct we solve a conjecture addressed in [10 by proving that any multiple of the Eguchi-Hanson metric on the blow-up of $\mathbb{C}^{2}$ at the origin is not projectively induced.
\end{abstract}

\section{INTRODUCTION}

It is still an open problem to classify those complex manifolds which admit a Ricci flat and projectively induced Kähler metric. Here a Kähler metric $g$ on a complex manifold $M$ (not necesseraly compact) is said to be projectively induced if there exists a Kähler (isometric and holomorphic) immersion of $(M, g)$ into the complex projective space $\left(\mathbb{C P}^{N}, g_{F S}\right), N \leqslant+\infty$, endowed with the Fubini-Study metric $g_{F S}$, namely the metric whose associated Kähler form is given in homogeneous coordinates by $\omega_{F S}=$ $\frac{i}{2 \pi} \partial \bar{\partial} \log \left(\left|Z_{0}\right|^{2}+\cdots+\left|Z_{N}\right|^{2}\right)$. This problem, which represents a special case of the classical and well-studied one dealing with Kähler-Einstein and projectively induced metrics, has been addressed in [10] where the authors proposed the following:

Conjecture: A Ricci-flat projectively induced metric is flat.

Roughly speaking the conjecture seems plausible since Ricci flat metrics are solutions of the Monge-Ampere equation and one should expect that solutions to such a nonlinear PDE would be "algebraic" (namely projectively induced) only in very special cases. In [10] the conjecture is proved for radial metrics by showing that the Eguchi-Hanson metric $g_{E H}$ on the blow-up of $\mathbb{C}^{2}$ at the origin is not projectively induced (cfr. [10, Cor. 3.3], see also [14] for not-radial cases). It is worth pointing out that Ricci-flatness, even in the radial case, cannot be weakened to scalar-flatness, as shown by the Simanca metric (cfr. [10, Th. 1.3], see also [3]).

2010 Mathematics Subject Classification. Primary 53C55; Secondary 58C25; 58F06.

Key words and phrases. Calabi's diastasis function, Ricci flat metric, projectively induced metric, flag manifold.

The first and the third authors were supported by Prin 2015 - Real and Complex Manifolds; Geometry, Topology and Harmonic Analysis - Italy, by GESTA - Funded by Fondazione di Sardegna and Regione Autonoma della Sardegna and by KASBA- Funded by Regione Autonoma della Sardegna.

Finally, all the three authors were supported by INdAM GNSAGA - Gruppo Nazionale per le Strutture Algebriche, Geometriche e le loro Applicazioni. 
Of course a necessary condition for a Kähler metric $g$ on a complex manifold $M$ to be projectively induced is that its associated Kähler form is integral, i.e. $[\omega] \in H^{2}(M, \mathbb{Z})$, where $[\omega]$ denotes the de-Rham class of $\omega$. Nevertheless, even if one is able to verify that a given Ricci flat metric $g$ with associated integral Kähler form $\omega$ is not projectively induced it is not an easy task to understand if the same is true for a (Ricci flat) metric homothetic to $g$, namely $\lambda g$. Indeed in the noncompact case, due for example to the fact that $\lambda \omega$ is always integral provided $M$ is contractible, the structure of the set of the positive real numbers $\lambda \in \mathbb{R}^{+}$for which $\lambda g$ is projectively induced is in general less trivial than in the compact case (where it is always discrete). For example if $\Omega$ is an irreducible bounded symmetric domain endowed with its Bergman metric $g_{B}$ one can prove (see [11]) that $\left(\Omega, \lambda g_{B}\right)$ admits a Kähler immersion into $\mathbb{C} P^{\infty}$ if and only if $\lambda$ belongs to the so called Wallach set of $\Omega$. Another interesting example is given by the Cigar metric $g=\frac{d z \otimes d \bar{z}}{1+|z|^{2}}$ on $\mathbb{C}$ which is not projectively induced together with all its multiples $\lambda g$ (cfr. [12]). The proof of this result is quite involved and it is based on some properties of Bell polynomials (see also Section 4 below).

The aim of this paper is to verify the validity of the above conjecture for the Ricci flat metrics on holomorphic line bundles over compact Kähler-Einstein manifolds constructed by Calabi in [5]. In order to state our main result (Theorem 10) we briefly recall Calabi's construction.

Let $(M, g)$ be a compact Kähler-Einstein manifold of complex dimension $n-1$ and with associated Kähler form $\omega_{g}$. Let $k_{0}>0$ be the Einstein constant of $g$, namely $\rho_{g}=k_{0} \omega_{g}$, being $\rho_{g}=-\frac{i}{\pi} \partial \bar{\partial} \log \operatorname{det}(g)$ the Ricci form. Let $\pi: \Lambda^{n-1} M \rightarrow M$ be the canonical line bundle over $M$, with hermitian metric $h$ given, for a system of local coordinates $z=\left(z_{1}, \ldots, z_{n-1}\right)$ on an open set $U \subset M$, by:

$$
h(\xi):=h\left(\xi d z_{1} \wedge \cdots \wedge d z_{n-1}, \xi d z_{1} \wedge \cdots \wedge d z_{n-1}\right)=\operatorname{det}(g)^{-1}|\xi|^{2} .
$$

Since the metric $g$ is assumed to be Kähler-Einstein one has:

$$
\frac{i}{\pi} \partial \bar{\partial} \log h(\xi)=k_{0} \omega_{g}
$$

Let $u:[0,+\infty) \rightarrow \mathbb{R}$ be the smooth function defined by:

$$
u(x)=\frac{n}{k_{0}}\left[(1+c x)^{\frac{1}{n}}-1\right]-\sum_{j=1}^{n-1} \frac{1-\tau^{j}}{k_{0}} \log \left[\frac{(1+c x)^{\frac{1}{n}}-\tau^{j}}{1-\tau^{j}}\right],
$$

where $c>0$ is constant and $\tau=e^{\frac{2 \pi i}{n}}$.

One easily verifies that the function $u$ satisfies:

(i) $1+k_{0} x u^{\prime}(x)>0, \quad u^{\prime}(x)+x u^{\prime \prime}(x)>0$,

(ii) $\left(1+k_{0} x u^{\prime}(x)\right)^{n-1}\left(u^{\prime}(x)+x u^{\prime \prime}(x)\right)=c$.

We have the following beautiful result due to Calabi.

Theorem C (Calabi [5]) Let $(M, g)$ be a compact Kähler-Einstein manifold of complex dimension $n-1$ and positive Einstein constant $k_{0}$ and $u$ the function given by (2). If $\Phi$ 
is a Kähler potential for $g$, i.e. $\omega_{g}=\frac{i}{2 \pi} \partial \bar{\partial} \Phi$ on $U$, then the function $\Psi: \pi^{-1}(U) \rightarrow \mathbb{R}$ defined by

$$
\Psi=\Phi \circ \pi+u\left(\operatorname{det}(g)^{-1}|\xi|^{2}\right)
$$

is a Kähler potential on $\pi^{-1}(U)$ for a Ricci flat and complete metric $g_{C}$ on the total space $\Lambda^{n-1} M$.

REMARK 1. Notice that if $g_{C}$ is the Ricci flat metric corresponding to $(M, g)$ as in the previous theorem, one easily deduces that $\alpha g_{C}, \alpha>0$, is the metric corresponding to $(M, \alpha g)$.

The previous theorem represents a special case of a more general construction due to Calabi himself (see [5] for more details). More precisely, Calabi considers a holomorphic hermitian line bundle $(L, h) \rightarrow M$ over a compact Kähler-Einstein manifold $(M, g)$, with Einstein constant $k_{0}$ (of arbitrary sign) such that

$$
\operatorname{Ric}(h)=-\ell \omega,
$$

for some real number $\ell$, where $\operatorname{Ric}(h)$ is the two-forms on $L$ given by:

$$
\operatorname{Ric}(h)=-\frac{i}{\pi} \partial \bar{\partial} \log h(\sigma(x), \sigma(x))
$$

for a trivializing holomorphic section $\sigma$ of $L$.

Then, Calabi shows that there exists a Kähler-Einstein metric $\tilde{g}$ with Einstein constant $k_{0}-\ell$ on an open subset $V$ of $L$, Moreover, when $\tilde{g}$ is Ricci flat, i.e. $\ell=k_{0}$, then $\tilde{g}$ is defined on the whole $L$, i.e. $V=L$. Notice also that $\tilde{g}$ is constructed in such a way that the natural inclusion $M \hookrightarrow L$ is a Kähler immersion, i.e. $\tilde{g}_{\mid M}=g$.

We can now state our main result.

Theorem 1. Let $(M, g)$ be a compact Kähler-Einstein manifold with Einstein constant $k_{0}$. The Calabi's metric $\tilde{g}$ on $L$ cannot be simultaneously Ricci flat and projectively induced.

When the manifold $(M, g)$ is assumed to be homogeneous, i.e. a flag manifold, Theorem 1 gives a large family of Ricci flat metrics which are not projectively induced (the reader is referred to 9] for an explicit description of the Kähler metrics and their potentials on flag manifolds). In particular, when $(M, g)=\left(\mathbb{C} P^{1}, g_{F S}\right)$ then the Calabi's Ricci flat metric $g_{C}$ on $O(-2)=\Lambda^{1} \mathbb{C} P^{1}$ is the celebrated Eguchi-Hanson metric $g_{E H}$ on the blow-up of $\mathbb{C}^{2}$ at the origin (see [5], [10] and references therein). Then, by Remark 1 one obtains the following corollary of Theorem 1, which thereby solves a question raised in [10].

Corollary 1. The metric $m g_{E H}$ is not projectively induced for any positive integer $m$.

The proof of Theorem 1 is given in Section 3. Roughly speaking we first show that if the metric $\tilde{g}$ on $L$ is projectively induced then $\tilde{g}=g_{C}$ (and $L=\Lambda^{n-1} M$ ). Then we use the structure of Calabi's diastasis function $D_{g_{C}}$ for the metric $g_{C}$ and its link with the diastasis 
of the metric $g$ (to whom Section 2 is dedicated) to show that $g_{C}$ is not projectively induced. Finally, in Section 4 we prove an inequality on Bell polynomials (Theorem 2) based on the fact that the metric $m g_{C}$ is not projectively induced. We were not able to find a direct proof of Theorem 2, Nevertheless, we believe that the interplay between Bell polynomials and projectively induced metrics should deserve further investigation.

\section{Calabi's diastasis funCtion for the Metric $g_{C}$}

Let $(X, G)$ be an $n$-dimensional real analytic Kähler manifold and denote by $\Omega$ the Kähler form associated to $G$. Set local coordinates $z=\left(z_{1}, \ldots, z_{n}\right)$ on a coordinate chart $U \subset X$ and denote by $\varphi: U \rightarrow \mathbb{R}$ a Kähler potential for $G$ on $U$, i.e. $\left.\Omega\right|_{U}=\frac{i}{2} \partial \bar{\partial} \varphi$. Calabi's diastasis function [2] for $G$ on $U$ is given by:

$$
D_{G}\left(z, z^{\prime}\right)=\tilde{\varphi}(z, \bar{z})+\tilde{\varphi}\left(z^{\prime}, \bar{z}^{\prime}\right)-\tilde{\varphi}\left(z, \bar{z}^{\prime}\right)-\tilde{\varphi}\left(z^{\prime}, \bar{z}\right),
$$

where $\tilde{\varphi}$ is the analytic continuation of $\varphi$ on a neighborhood of the diagonal of $U \times U$. It is easy to see that the diastasis is symmetric in $z, z^{\prime}$ and that, once fixed one of its two entries, it is a Kähler potential for $G$. In particular, we will denote by $D_{G}(z):=D_{G}(0, z)$ the diastasis centered at the origin of the coordinate system. Among the other Kähler potentials, the diastasis function is characterized by the fact that in every coordinate system $(z)$ centered in $p$, the $\infty \times \infty$ matrix of coefficients $\left(a_{j k}\right)$ in its power expansion in terms of $z$ and $\bar{z}$ around the origin:

$$
D_{G}(z)=\sum_{j, k=0}^{\infty} a_{j k} z^{m_{j}} \bar{z}^{m_{k}},
$$

satisfies $a_{j 0}=a_{0 j}=0$ for every nonnegative integer $j$, i.e it does not contain either holomorphic or antiholomorphic terms. In the multi-index notation the $m_{j}$ 's are $n$-tuples of integers arranged in lexicographic order.

In order to prove Theorem 1 we recall Calabi's criterion for a Kähler metric to admit a local Kähler immersion into a finite or infinite dimensional complex projective space $\left(\mathbb{C P}^{N}, g_{F S}\right), N \leqslant \infty$, through the diastasis function (the reader is referred to [13] for a more detailed and updated exposition of the subject).

Lemma 1. (Calabi's criterion) Let $(X, G)$ be a real analytic Kähler manifold and let $D_{G}$ be its diastasis function around the origin. Then $(X, G)$ admits a local Kähler immersion into $\left(\mathbb{C} P^{N}, g_{F S}\right)$ if and only if the $\infty \times \infty$ matrix of coefficients $\left(b_{j k}\right)$ in the power expansion with respect to $z$ and $\bar{z}$ :

$$
e^{D_{G}(z)}-1=\sum_{j, k=0}^{\infty} b_{j k} z^{m_{j}} \bar{z}^{m_{k}},
$$

is positive semidefinite.

In the following lemma we describe Calabi's diastasis function for the metric $g_{C}$ on $\Lambda^{n-1} M$ given in Theorem C. In order to do that we need to introduce the concept of 
Bochner's coordinates (cfr. 4], 2]). Given a real analytic Kähler metric $G$ on $X$ and a point $p \in X$, one can always find local (complex) coordinates in a neighborhood of $p$ such that

$$
D_{G}(z)=|z|^{2}+\sum_{|j|,|k| \geqslant 2} a_{j k} z^{j} \bar{z}^{k}
$$

These coordinates, uniquely defined up to a unitary transformation, are called the Bochner's coordinates with respect to the point $p$.

Lemma 2. Let $(M, g)$ be a compact Kähler-Einstein manifold with positive Einsten constant $k_{0}$ and $D=D_{g}: U \rightarrow \mathbb{R}$ be its diastasis function centered at the origin. Then the diastasis function for the metric $g_{C}$ in Bochner's coordinates reads as:

$$
D_{g_{C}}=D+u\left(e^{\frac{k_{0}}{2} D}|\xi|^{2}\right) .
$$

Moreover, the matrix of coefficients $\left(b_{i l}\right)$ given on (77) associated to $D_{g_{C}}$ is a block matrix where each block $\left(b_{j k}^{r}\right), r=0,1,2, \ldots$, is given by

$$
\left(b_{j k}^{r}\right)=h_{r}(u)\left(c_{j k}^{r}\right),
$$

where $\left(c_{j k}^{r}\right)$ is the matrix of coefficients (7) associated to $\left(r \frac{k_{0}}{2}+1\right) D$ and $h_{r}(u)$ is a constant that depends on the derivatives of $u(x)$ evaluated at $x=0$ up to the $r$-th order. In particular:

$$
\begin{gathered}
h_{1}(u)=u^{\prime}(0) ; \\
h_{2}(u)=\frac{1}{2}\left(u^{\prime}(0)^{2}+u^{\prime \prime}(0)\right) ; \\
h_{3}(u)=\frac{1}{6}\left(u^{\prime}(0)^{3}+3 u^{\prime \prime}(0) u^{\prime}(0)+u^{\prime \prime \prime}(0)\right) ; \\
h_{4}(u)=\frac{1}{24}\left(u^{\prime}(0)^{4}+6 u^{\prime \prime}(0) u^{\prime}(0)^{2}+4 u^{\prime \prime \prime}(0) u^{\prime}(0)+3 u^{\prime \prime}(0)^{2}+u^{(i v)}(0)\right) .
\end{gathered}
$$

Proof. Let $z=\left(z_{1}, \ldots, z_{n-1}\right)$ be Bochner coordinates on a open set of $U \subset M$. The condition $\rho_{g}=k_{0} \omega_{g}$ reads:

$$
-i \partial \bar{\partial} \log \operatorname{det}\left(\frac{\partial^{2} D}{\partial z_{i} \partial \bar{z}_{j}}\right)=\frac{k_{0}}{2} i \partial \bar{\partial} D
$$

that is:

$$
\log \operatorname{det}\left(\frac{\partial^{2} D}{\partial z_{i} \partial \bar{z}_{j}}\right)=-\frac{k_{0}}{2} D+f+\bar{f}
$$

where $f$ is a holomorphic function on $U$. By using the condition that $z$ are Bochner coordinates and $D$ is the diastasis, it is not hard to see (cfr. also [1]) that $f=0$, and hence we can write:

$$
\operatorname{det}(g)=\operatorname{det}\left(\frac{\partial^{2} D}{\partial z_{i} \partial \bar{z}_{j}}\right)=e^{-\frac{k_{0}}{2} D} .
$$

By (3) it follows that (8) is a Kähler potential for $g_{C}$ on $\pi^{-1}(U)$. Moreover, since the expansion (6) of $D$ does not contain holomorphic or antiholomorphic terms one easily 
sees that the same is true for the expansion of $D_{g_{C}}$ with respect to the coordinates $z_{1}, \ldots, z_{n-1}, \xi$.

In order to prove the second assertion observe first that since $D_{g_{C}}$ depends on $\xi, \bar{\xi}$ only through $|\xi|^{2}$, all the derivatives taken a different number of times with respect to $\xi$ than $\bar{\xi}$, vanish once evaluated at $\xi=0$. Thus, the nonzero entries of $\left(b_{i l}\right)$ are of the form:

$$
\frac{1}{m_{j} ! m_{k} !} \frac{\partial^{\left|m_{j}\right|+\left|m_{k}\right|}}{\partial z^{m_{j}} \partial \bar{z}^{m_{k}}}\left[\left.\frac{1}{r !^{2}} \frac{\partial^{2 r}}{\partial \xi^{r} \partial \bar{\xi}^{r}}\left(e^{D+u\left(e^{\frac{k_{0}}{2} D}|\xi|^{2}\right)}-1\right)\right|_{\xi=0}\right]_{z=\bar{z}=0},
$$

and, for each $r=0,1,2, \ldots$, we have a block submatrix $\left(b_{j k}^{r}\right)$ of $\left(b_{i l}\right)$ defined by:

$$
\begin{aligned}
b_{j k}^{r} & :=\frac{1}{m_{j} ! m_{k} !} \frac{\partial^{\left|m_{j}\right|+\left|m_{k}\right|}}{\partial z^{m_{j}} \partial \bar{z}^{m_{k}}}\left[\left.\frac{1}{r !^{2}} \frac{\partial^{2 r}}{\partial \xi^{r} \partial \bar{\xi}^{r}}\left(e^{D+u\left(e^{\frac{k_{0}}{2} D}|\xi|^{2}\right)}-1\right)\right|_{\xi=0}\right]_{z=\bar{z}=0} \\
& =\left.h_{r}(u) \frac{1}{m_{j} ! m_{k} !} \frac{\partial^{\left|m_{j}\right|+\left|m_{k}\right|}}{\partial z^{m_{j}} \partial \bar{z}^{m_{k}}} e^{\left(r \frac{k_{0}}{2}+1\right) D}\right|_{z=\bar{z}=0} \\
& =h_{r}(u) c_{j k}^{r}
\end{aligned}
$$

where:

$$
c_{j k}^{r}:=\left.\frac{1}{m_{j} ! m_{k} !} \frac{\partial^{\left|m_{j}\right|+\left|m_{k}\right|}}{\partial z^{m_{j}} \partial \bar{z}^{m_{k}}} e^{\left(r \frac{k_{0}}{2}+1\right) D}\right|_{z=\bar{z}=0},
$$

and $h_{r}(u)$ is a constant that depends on the derivatives up to $r$-th order on $u(x)$. Thus (9) is proved. Finally, (10), (11), (12) and (13) follows by straightforward computations.

\section{Proof of Theorem 1}

Let us begin with the following lemma.

Lemma 3. Let $(M, g)$ be a compact Kähler-Einstein manifold with Einstein constant $k_{0}$. If the Ricci flat Calabi's metric $g_{C}$ on $\Lambda^{n-1} M$ is projectively induced then the following conditions hold true.

(a) $\frac{k_{0}}{2}$ is a positive integer;

(b) $\left(r \frac{k_{0}}{2}+1\right) g$ is projectively induced, for any $r=0,1 \ldots$;

(c) each $h_{r}(u)$ given by (9) is non negative, for any $r=0,1 \ldots$

Proof. The assumption that $g_{C}$ is projectively induced and Calabi's criterion (Lemma 1) imply that the block submatrices $\left(b_{j k}^{r}\right)=h_{r}(u)\left(c_{j k}^{r}\right)$ given by (9) are positive semidefinite, for any $r=0,1 \ldots$ Since $g_{C \mid M}=g$ it follows that also $g$ is projectively induced, and so (b) is valid for $r=0$. When $r=1$ ( $\operatorname{cfr}$. (10) $), h_{1}(u)=u^{\prime}(0) e^{u(0)}=c>0$ (where $c$ is the constant appearing in $(i i)$ before Theorem $\mathrm{C}$ ) which proves that $\left(c_{j k}^{1}\right)$ is positive semidefinite. Again by Calabi's criterion one deduces that $\left(\frac{k_{0}}{2}+1\right) g$ is projectively induced. If $\omega$ is the Kähler form associated to $g$ the fact that both $g$ and $\left(\frac{k_{0}}{2}+1\right) g$ are projectively induced imply that $\omega$ and $\left(\frac{k_{0}}{2}+1\right) \omega$ are integral forms, forcing $\frac{k_{0}}{2}$ to be a positive integer, i.e. (a). Hence $\left(r \frac{k_{0}}{2}+1\right)$ is a positive integer and (b) follows by composing the Kähler immersion inducing $g$ with a suitable normalization of the Veronese embbedding (cfr. [2, Theorem 13]). Finally, by combining (b), (9) and Calabi's criterion we get (c). 
Proof of Theorem 1. We start noticing that if the metric $\tilde{g}$ on $L$ is Ricci flat and projectively induced then $\tilde{g}=g_{C}$ and $L=\Lambda^{n-1} M$. Indeed, since $\tilde{g}_{\mid M}=g$ one has that also $g$ is projectively induced and, by a result of Hulin [7] $(M, g)$ has positive Einstein constant $k_{0}$. Therefore, $M$ is simply-connected, and the Ricci flatness of $\tilde{g}$, i.e $\ell=k_{0}$, combined with (4) yields

$$
c_{1}(L)=\left[-\frac{i}{2 \pi} \partial \bar{\partial} \log h\right]=-\frac{k_{0}}{2}[\omega]=-\left[\frac{\rho}{2}\right]=c_{1}\left(\Lambda^{n-1} M\right),
$$

and so $L$ is holomorphically equivalent to the canonical bundle $\Lambda^{n-1} M$.

Thus, assume by contradiction that the metric $g_{C}$ is projectively induced. We will show that the sign of some $h_{r}(u)$ is negative and by (c) of Lemma 3 this gives the desired contradiction. Setting $x=0$ in equation (ii) before Theorem $\mathrm{C}$, one has $u^{\prime}(0)=c$, while differentiating the same equation with respect to $x$ one gets:

$$
(n-1) k_{0}\left(1+k_{0} x u^{\prime}\right)^{n-2}\left(u^{\prime}+x u^{\prime \prime}\right)^{2}+\left(1+k_{0} x u^{\prime}\right)^{n-1}\left(2 u^{\prime \prime}+x u^{\prime \prime \prime}\right)=0
$$

which, evaluated in $x=0$, gives:

$$
u^{\prime \prime}(0)=-\frac{(n-1) k_{0}}{2} u^{\prime}(0)^{2}
$$

Combining this with $u^{\prime}(0)=c$, one deduces:

$$
u^{\prime \prime}(0)+u^{\prime 2}(0)=\left(1-\frac{(n-1) k_{0}}{2}\right) c^{2} .
$$

Recalling that $c>0$ and that, by (a) of Lemma $3, \frac{k_{0}}{2}$ is a positive integer, we deduce by (11) that $h_{2}(u)$ is negative except for $k_{0}=2$ and $n=2$ (where it vanishes). For this values we will show that $h_{4}(u)<0$. In order to do that, we differentiate (14) twice and evaluating at $u^{\prime}(0)=c$ and $u^{\prime \prime}(0)=-c^{2}$ we get $u^{\prime \prime \prime}(0)=4 c^{3}$, and $u^{(i v)}(0)=-30 c^{4}$. Plugging these into (13) gives that $h_{4}(u)=-\frac{2}{3} c^{4}<0$, and we are dond. 1 .

REMARK 2. It is an open problem to classify the compact Kähler-Einstein manifolds admitting Kähler immersions into complex projective spaces. The only known examples are indeed flag manifolds. The reader is referred to [13, Ch.3] for other properties of projectively induced homogeneous metrics and, in particular, for the proof that any integral Kähler form on a compact flag manifold is projectively induced. Combining this result with the fact that on any flag manifold one can find a Kähler-Einstein integral form with Einstein constant $k_{0}=2$ (see [8]) we deduce the existence of a projectively induced Kähler-Einstein metric satisfying (a) in Lemma 3. Actually by taking the Kähler product of such manifolds one can construct Kähler-Einstein metrics with arbitrary large even value of their Einstein constant. This means that also if one restricts to the case of flag manifolds the proof of Theorem 1 cannot be deduced by simply showing that condition (a) is not satisfied.

\footnotetext{
${ }^{1}$ The reason why we look at the coefficient $h_{4}(u)$ is because by (12) one deduces $h_{3}(u)=\frac{c^{3}}{3}>0$.
} 


\section{An application to A nUmerical PRoblem}

Recall that the partial (exponential) Bell polynomials $B_{r, j}(x):=B_{r, j}\left(x_{1}, \ldots, x_{r-j+1}\right)$ of degree $r$ and weight $j$ are defined by (see e.g. [6, p. 133]):

$$
B_{r, j}\left(x_{1}, \ldots, x_{r-j+1}\right)=\sum \frac{r !}{s_{1} ! \cdots s_{r-j+1} !}\left(\frac{x_{1}}{1 !}\right)^{s_{1}}\left(\frac{x_{2}}{2 !}\right)^{s_{2}} \cdots\left(\frac{x_{r-j+1}}{(r-j+1) !}\right)^{s_{r-j+1}}
$$

where the sum is taken over the integers solutions of:

$$
\left\{\begin{array}{l}
s_{1}+2 s_{2}+\cdots+j s_{r-j+1}=r \\
s_{1}+\cdots+s_{r-j+1}=j
\end{array}\right.
$$

The complete Bell polynomials are given by:

$$
Y_{r}\left(x_{1}, \ldots, x_{r}\right)=\sum_{j=1}^{r} B_{r, j}(x), \quad Y_{0}:=0,
$$

and the role they play in our context is given by the following formula [6, Eq. 3b, p.134]:

$$
\left.\frac{d^{r}}{d x^{r}}\left(\exp \left(\sum_{k=1}^{\infty} a_{k} \frac{x^{k}}{k !}\right)\right)\right|_{0}=Y_{r}\left(a_{1}, \ldots, a_{r}\right) .
$$

We can prove the following inequality.

Theorem 2. Let $n \in \mathbb{Z}^{+}, n \geqslant 2$. For any $q \in \mathbb{Q}^{+}$, there exists $r$ sufficiently large such that the following inequality holds true:

$$
(-1)^{r} \sum_{j=1}^{r}(-q)^{j} B_{r, j}\left(1, \frac{n-1}{2}, \frac{(n-1)(2 n-1)}{3}, \ldots, \frac{1}{l} \prod_{s=1}^{l-1}(n s-1), \ldots\right)<0 .
$$

Proof. Let $u(x)$ be the function given by (2). Then a straightforward computation by differentiating (ii) before Theorem $\mathrm{C}$, shows that its series expansion is given by:

$$
u(x)=\sum_{k=1}^{\infty} \frac{a_{k}}{k !} x^{k}, \quad a_{1}=c,
$$

where:

$$
a_{j}=\frac{(-1)^{j+1}}{j} c^{j} k_{0}^{j-1} \prod_{s=1}^{j-1}(n s-1), \quad j=2,3,4, \ldots .
$$

Let $m>0$. By (17) it is easy to see that:

$$
\left.\frac{\partial^{2 r}}{\partial \xi^{r} \partial \bar{\xi}^{r}}\left[e^{m D+m u\left(e^{\frac{k_{0}}{2} D}|\xi|^{2}\right)}-1\right]\right|_{\xi=0}=r ! e^{\left(r \frac{k_{0}}{2}+m\right) D} \sum_{j=1}^{r} m^{j} B_{r, j}\left(a_{1}, a_{2}, \ldots\right),
$$

and in particular, the block matrices described in (9) are now given by:

$$
\left(b_{j k}^{r, m}\right)=h_{r}(u, m)\left(c_{j k}^{r, m}\right),
$$


where:

$$
h_{r}(u, m):=r ! \sum_{j=1}^{r} m^{j} B_{r, j}\left(a_{1}, a_{2}, \ldots\right), \quad c_{j k}^{r, m}:=\left.\frac{1}{m_{j} ! m_{k} !} \frac{\partial^{\left|m_{j}\right|+\left|m_{k}\right|}}{\partial z^{m_{j}} \partial \bar{z}^{m_{k}}} e^{\left(r \frac{k_{0}}{2}+m\right) D}\right|_{z=\bar{z}=0},
$$

(in this notations, the constants $h_{r}(u)$ appearing in (9) are given by $h_{r}(u)=h_{r}(u, 1)=$ $\left.r ! Y_{r}\left(a_{1}, \ldots, a_{r}\right)\right)$. By Theorem 1 and Remark 1 the metric $m g_{C}$ on $\Lambda^{n-1} M$ is not projectively induced for any $m>0$. By Calabi's criterion this implies that each block $\left(b_{j k}^{r, m}\right)$ is not semipositive definite. If we assume $(M, g)$ to be projectively induced and $r \frac{k_{0}}{2}+m$ to be a positive integer for any positive integer $r$, i.e. $\frac{k_{0}}{2}, m \in \mathbb{Z}^{+}$, then Calabi's criterion applied to $\left(M,\left(r \frac{k_{0}}{2}+m\right) g\right)$ implies that each block $\left(c_{j k}^{r, m}\right)$ is semipositive definite. Thus, at least one of the constants $h_{r}(u, m)$ is forced to be negative, i.e. for any $m \in \mathbb{Z}^{+}$there exists a sufficiently large $r$ such that:

$$
\sum_{j=1}^{r} m^{j} B_{r, j}\left(a_{1}, a_{2}, \ldots\right)<0
$$

Since:

$$
B_{r, j}\left(t p x_{1}, t p^{2} x_{2}, \ldots, t p^{r-j+1} x_{r-j+1}\right)=t^{j} p^{r} B_{r, j}\left(x_{1}, \ldots, x_{r-j+1}\right),
$$

by (19) we can write:

$$
\begin{aligned}
\sum_{j=1}^{r} m^{j} B_{r, j}\left(a_{1}, a_{2}, \ldots\right) & =\left(-c k_{0}\right)^{r} \sum_{j=1}^{r}\left(-\frac{m}{k_{0}}\right)^{j} . \\
\cdot & B_{r, j}\left(1, \frac{n-1}{2}, \frac{(n-1)(2 n-1)}{3}, \ldots, \frac{1}{l} \prod_{s=1}^{l-1}(n s-1), \ldots\right) .
\end{aligned}
$$

At this point, (18) follows by observing that one can construct examples of projectively induced Kähler-Einstein manifolds with any positive integer value of $k_{0} / 2$ (see Remark 2).

\section{REFERENCES}

[1] C. Arezzo, A. Loi and F. Zuddas, Some remarks on the symplectic and Kähler geometry of toric varieties, AMPA 4 (2016), 1287-1304.

[2] E. Calabi, Isometric Imbedding of Complex Manifolds, Ann. of Math. 58 (1953), 1-23.

[3] F. Cannas Aghedu and A. Loi, The Simanca metric admits a regular quantization, Ann. Global Anal. Geom. 56 (2019), no. 3, 583-596.

[4] S. Bochner, Curvature in Hermitian metric. Bull. Amer. Math. Soc. 53 (1947), 179-195.

[5] E. Calabi, Métriques kählériennes et fibrés holomorphes. (French) [Kähler metrics and holomorphic vector bundles], Ann. Sci. École Norm. Sup. (4) 12 (1979), no. 2, 269-294.

[6] L. Comtet, Advanced combinatorics, the art of the finite and infinite expansion, Springer, 1974.

[7] D. Hulin, Kähler-Einstein metrics and projective embeddings, J. Geom. Anal. 10 (2000), 525528.

[8] A. Loi, R. Mossa and F. Zuddas, The log-term of the disc bundle over a homogeneous Hodge manifold, Ann. Global Anal. Geom. 51 (2017), no. 1, 35-51. 
[9] A. Loi, R. Mossa and F. Zuddas, Bochner coordinates on flag manifolds, Bull. Braz. Math. Soc. (N.S.) 50 (2019), no. 2, 497-514.

[10] A. Loi, F. Salis and F. Zuddas, Two conjectures on Ricci-flat Kähler metrics, Math. Z. 290 (2018), 599-613.

[11] A. Loi and M. Zedda, Kähler-Einstein submanifolds of the infinite dimensional projective space, Math. Ann. 350 (2011), 145-154.

[12] A. Loi and M. Zedda, On Calabi's diastasis function of the Cigar metric, J. Geom. Phys. 110 (2016), 269-276.

[13] A. Loi and M. Zedda, Kähler Immersions of Kähler Manifolds into Complex Space Forms, Lecture Notes of the Unione Matematica Italiana 23, Springer, 2018.

[14] M. Zedda, A note on projectively induced Ricci-flat Kähler metrics, preprint (2019), arXiv:1901.03129 [math.DG].

(Andrea Loi, Fabio Zuddas) Dipartimento di Matematica e Informatica, Università di Cagliari, Via Ospedale 72, 09124 Cagliari, Italy

E-mail address: loi@unica.it, fabio.zuddas@unica.it

(Michela Zedda) Dipartimento di Scienze Matematiche, Fisiche e Naturali, Parco Area delle Scienze 53/A Parma (Italy)

E-mail address: michela.zedda@unipr.it 